\title{
Global Stability for Distributed Systems with Changing Contact States
}

\author{
T. D. Murphey, J. W. Burdick \\ Engineering and Applied Science, California Institute of Technology \\ Mail Code 104-44, Pasadena, CA 91125 USA \\ \{murphey,jwb\}@robotics.caltech.edu
}

\begin{abstract}
This paper analyzes the global stability of distributed manipulation control schemes. The "programmable vector field" approach, which assumes that the system's control actions can be approximated by a continous vector force field, is a commonly proposed scheme for distributed manipulation control. In practical implementations, the continuous control force field idealization must then be adapted to the specifics of the discrete physical actuator array. However, in Murphey and Burdick [2001] it was shown that when one takes into account the discreteness of actuator arrays and realistic models of the actuator/object contact mechanics, the controls designed by the continuous approximation approach can be unstable at the desired equilibrium configuration. We introduced a discontinuous feedback law that locally stabilizes the manipulated object at the equilibrium. However, the stability of this feedback law only holds in a neighborhood of the equilibrium. In this paper we show how to combine the programmable vector field approach and our local feedback stabilization law to achieve a globally stable distributed manipulation control system. Simulations illustrate the method.
\end{abstract}

\section{Introduction and Previous Work}

A distributed manipulation system consists of an (roughly planar) array of actuators that can re-position an object by the movements of its array elements. In the future, arrays of this type should be useful for industrial assembly operations where parts must be robustly transported and precisely positioned. This paper considers the global behavior of distributed actuator control systems. We present a method for globally stabilizing a part on a set of actuators in the plane, and prove the global stability of this method.

Methods to design distributed manipulation control systems have been proposed in several works, including Erdmann and Mason [1988] and Goldberg [1993]. A common approach is based on the notion of programmable vector fields. This method was first pioneered in Böhringer et al. [1997]. In this methodology, one makes the possibly unrealistic assumption that the array's control capability can be idealized as a continuous distribution of forces across the array surface. In this abstraction, the manipulated object moves under the influence of these continuously distributed forces. The control design problem reduces to the selection of a continuous force field distribution that will locally transport the object to a prescribed position, and then stabilize it at that configuration. The basic control strategy is to choose a sequence of force fields which move the part from one equilibrium to another equilibrium. To implement the control strategy on the real array, one must adapt the continuous vector field control to the real (and discrete) actuator array. For a good description of this approach, see Böhringer et al. [2000].

This approach is experimentally known to work in MEMS-fabricated actuator arrays, where the array elements are "small" and "close" together relative to the object being manipulated Böhringer et al. [2000]. However, in cases where the actuators are far apart (i.e., the continuous actuation approximation is poor) or the coefficient of friction $\mu$ is very high, the continuous approximation is known not to work as well (see Murphey and Burdick [2001]). In these cases, the continuous approximation does not adequately incorporate the discrete nature of the actual array and the contact mechanics of the object/array interface. In Murphey and Burdick [2001] we showed that when one uses the programmable vector field method, these effects will lead to instability in the orientation $(\theta)$ component of the moving object's $S E(2)$ location. We then introduced a local feedback law that stabilizes the object at the equilibrium.

While Murphey and Burdick [2001] considered the local stabilizability and control of the moving object near its equilibrium configuration, in this paper we consider the global stabilizability and control synthesis problem. I.e., we consider the properties of the control system that are involved in guiding the object from any initial configuration to a desired equilibrium state. In particular, we present a way of blending the philosophies of Böhringer et al. [2000] and of Murphey and Burdick [2001] to produce a globally stabilizing control which only requires local feedback near an equilibrium point. The requirement that feedback need only be used near the equilibrium leads to economical implementations.

Section 2 provides the basic mathematical background required for the analysis and an overview of both previous results and of the modeling methodologies used. Section 3 has the main theorem of this paper, along with a discussion of how it applies to the special case of distributed manipulation. Section 4 presents illustrative simulation results. 


\section{Background}

This section provides background in both the theory of programmable vector fields and in the theory of nonsmooth vector fields. We additionally present the basics of the power dissipation method (PDM) for determining models of over-constrained systems that are moving quasi-statically. The PDM method was previously developed by the authors in Murphey and Burdick [2000]

\subsection{Programmable Force Fields}

The use of programmable vector fields for distributed manipulator control is based on a continuous "force field" abstraction which assumes that at each point on the manipulation surface one can specify the manipulation force at that point. The dynamics of the moving object are obtained by integrating the continuous force field to get a total force on the part. To use the controls on an actual array, where the manipulation forces will be generated at discrete points, one must adapt the continuous approximation to the geometry of a given discrete array. For a good reference, see Böhringer et al. [2000].

The most basic control law comes from the idea of a "squeeze" field. Squeeze fields are in general of the form $F=\{-\alpha x,-\beta y\}$ where $\alpha$ and $\beta$ are coefficients to be chosen by the control designer. These open loop control can stabilize an object to one of two stable equilibria. Using this idea as a basis, significant work has been done to produce unique equilibria for parts with no symmetry. Further improvements were made regarding computing the programmable force field to produce a unique stable equilibrium in Sudsang and Kavraki [2001]. These forces can be integrated over the body's surface to obtain the object's dynamical response. Mathematically, this translates as follows: we assume the part $w$ can be described by support characteristic function $w(x, y)$ where $w(x, y)$ is 1 everywhere on the object surface and 0 otherwise. Moreover, let the part be subject to a force field $f(x, y): \mathbb{R}^{2} \rightarrow \mathbb{R}^{2}$. Lastly, we make the reference frame of $w$ be at the center of mass, i.e.

$$
\int_{\mathrm{R}^{2}} p \omega(p) d p=0
$$

When the object lies at configuration $q=(x, y, \theta)$ the net force and torque on the object are:

$$
\begin{aligned}
F & =\int_{\mathbb{R}^{2}} p \omega(p) f\left(A_{\theta} p+t\right) d p \\
M & =\int_{\mathbb{R}^{2}} p \omega(p) A_{\theta} p \times f\left(A_{\theta} p+t\right) d p
\end{aligned}
$$

with $t=(x, y)^{r}$ and $A$ the $2 \times 2$ rotation matrix of angle $\theta$. The condition for stability is $F=M=0$. However, in cases where the actuators are far apart or the coefficient of friction $\mu$ is very high the continuous approximation is known not to work as well, because the objects being moved have dramatically different dynamics depending on the contact state. In Murphey and Burdick [2001] we showed that rotational instability will result from the switching of contact states that arises from the contact mechanics and the discreteness of the actuator array.

Remark: It should be noted that although the inputs for the programmable force field are forces and the inputs we are going to consider are vector field inputs (velocities), these two are in reality often the same set of inputs because the forces are typically generated by the friction caused by the wheel slipping at a given velocity. I.e. $F=-\mu N v$ where $\mu$ is the coefficient of friction, $N$ is the normal force, and $v$ is the velocity. Therefore the input classes are actually equivalent.

\subsection{The Power Dissipation Model}

To analyze control system performance, we seek models for distributed actuation systems that faithfully capture the system's essential physics, and that are tractable and amenable to control and motion planning analysis. In pursuit of this goal, we use a "power dissipation model" (PDM) approach to model the governing dynamics of a discrete actuator/object system. This method typically produces unique models that are relatively easy to obtain, and to which one can apply control system analysis methods. Since the method is a quasi-static modeling method, it produces first-order governing equations, instead of second order equations that are associated with Lagrange's equations. The primary disadvantage is that the method only applies to quasi-static systems. In Murphey and Burdick [2001] we argue that this assumption is quite good for our problems of interest, and for distributed manipulation arrays in general. Here we present an overview of the modeling methodology, and refer the reader to Murphey and Burdick [2001] and Murphey and Burdick [2000] for more details.

Let $q$ denote the configuration of the array/object system, consisting of the object's planar location, and the variables that describe the state of each actuator array element. Let us assume that the motion of the actuator array's variables are known. It is not hard to show that the relative motion of each contact between the object and an actuator array element can be modeled in the form $\omega(q) \dot{q}$. If $\omega(q) \dot{q}=0$, then the contact is not slipping (i.e., it is nonholonomic), while if $\omega(q) \dot{q} \neq 0$, then $\omega(q) \dot{q}$ describes the slipping velocity. In general, the moving object will be in contact with the actuator array at many points. In general, from kinematic considerations, one or more of the contact points must be in a slipping state. The power dissipation function measures the object's total energy dissipation due to contact slippage.

Definition 1 The Dissipation or Friction Functional for an $n$-contact state is defined to be

$$
\mathcal{D}=\sum_{i=1}^{n} \alpha_{i}|\omega(q) \dot{q}|
$$

where $\alpha_{i}=\mu_{i} N_{i}$, with $\mu_{i}$ and $N_{i}$ being the Coulomb friction coefficient and normal force at the $i^{\text {th }}$ contact, which are assumed known. 
We assume throughout that the object's contact with the manipulating surface is governed by the Coulomb friction law at each point of contact. We treat the object and the array element contact as a rigid body contact system (though approximate compliance effects can be easily incorporated into this paradigm). Let us assume without loss of generality that the object is in contact with several array elements. Let us further assume that the contact with each array element is a point contact, or set of point contacts (complex contacts such as line contacts are modeled as a set of point contacts in this approach). With sufficiently many contacts between the object and the manipulating surface, it will often be true that one or more contacts must slip during object motion, thereby dissipating energy. I.e., no motion exists where all of the contacts can be simultaneously slipless. These ideas lead to the following formal statement.

Power Dissipation Principle: With $\dot{q}$ small, an object's motion at any given instant is the one that minimizes $\mathcal{D}$.

The power dissipation method assumes that the object's motion at any given instant is the one that instantaneously minimizes power dissipation due to contact slippage. This method is adapted from the work of Alexander and Maddocks Alexander and Maddocks [1989] on wheeled vehicles. Here we briefly summarize some of the PDM's formal characteristics, which were first developed in the context of wheeled vehicles (Murphey and Burdick [2000]). In particular, we show that the power dissipation approach generically leads to multi-model (or hybrid) systems. For the omitted proofs, a greater discussion of these results, and a discussion of the relationship between the PDM method and the full Lagrangian for such a system, see Murphey and Burdick [2000].

We first consider the extent to which the function $\mathcal{D}$ having a unique minimum (and therefore a uniquely defined set of equations governing the object's motion) is generic.

Proposition 1 (Murphey and Burdick [2000]) Assume $\mathcal{D}$ is of the form in Definition 1 and that the $\mu$ is measurable in $x$ and $t$. Then the dissipation functional $\mathcal{D}$ has a unique minimum almost always (i.e. except on a set of measure 0)

That is, the PDM will almost always lead to a unique set of governing equations. Even in the non-generic case where the minimum of $\mathcal{D}$ is not unique, we have the following.

Proposition 2 (Murphey and Burdick [2000]) If $\dot{q}_{1}$ and $\dot{q}_{2}$ both minimize the dissipation functional found in Definition 1 , then so does $\operatorname{co}\left\{\dot{q}_{1}, \dot{q}_{2}\right\}$.

This result formalizes the intuition that if the power dissipated is equal for two velocities $\dot{q}_{i}$, then all possible trajectories whose velocity lies in the convex hull of the $\dot{q}_{i}$ will satisfy the minimum also. I.e., in the non-generic case when $\mathcal{D}$ does not have a unique minimum, we can still bound the object's motion. Our next definition describes the kind of system one obtains by using the PDM approach.
Definition 2 A system is a multi-model driftless affine system (MMDA) ${ }^{1}$ termed these if it can be expressed in the form

$$
\dot{x}=f_{\sigma_{1}}(x) u_{1}+f_{\sigma_{2}}(x) u_{2}+\cdots+f_{\sigma_{n}}(x) u_{n}
$$

where for any $x$ and $t, f_{\sigma_{i}}(x) \in\left\{g_{\alpha_{i}}(x) \mid \alpha_{i} \in I_{i}\right\}$, with $I_{i}$ an index set and $f_{i}$ measurable in $(x, t)$ and $g_{i}$ analytic in $(x, t)$ for all $i$.

An MMDA is a driftless affine nonlinear control system where each control vector fields may "switch" back and forth between different elements of a finite set. In our case, this switching corresponds to the switching between different contact states between the object and the array surface elements (i.e., different sets of slipping contacts) due to variations in contact geometry and surface friction properties. In Murphey and Burdick [2000] it was shown that the PDM generically leads to MMDA systems as in Definition 2.

Remark: Here we should comment on the relationship between the philosophies of the PDM approach and the programmable force field approach. The programmable force field method effectively assumes that there are an infinite number of actuators, that all of the actuators are slipping all the time, and that the physics of contact between the array surface and the object is not that important. Hence, the programmable force field method is more appropriate to gross motions where accuracy is less important and simplicity of analysis and the design problem is appealing. The PDM assumes that there is generally a finite number of discrete contact points, and incorporates Coulomb friction contact physics into the model. However, it is formally only applicable to quasi-static motions. As we argue in Murphey and Burdick [2001], the quasi-static assumption is quite good in general for distributed actuator arrays, and is a particularly good assumption near the object's equilibrium state. Therefore, the PDM is more appropriate to the analysis and control of local, quasi-static motions, near the equilibrium. These contrasting features inspire the merging of these two techniques in this paper.

2.3 Modeling the Equilibrium Point of a Programmable Vector Field

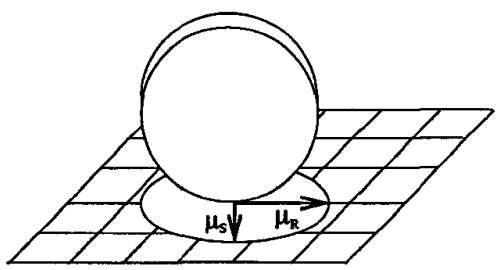

Figure 1: A wheel with anisotropic friction

Our PDM modeling approach is applicable to a wide variety of interfaces between the moving object and the array

\footnotetext{
${ }^{1}$ By abuse of notatation, in Murphey and Burdick [2000, 2001] we referred to these systems as "switched driftless affine systems". The nomenclature used here adheres to the most commonly used nomenclature in the control system literature
} 
actuators: rotating wheels, moving fingertips, sliding surfaces, etc. We only assume that local contact between the object and the actuator array is significantly influenced by Coulomb friction. Generally, there is no reason to believe that friction at each contact point will be uniform in all directions of the contact plane. Rather, we allow a smooth distribution of coefficient of friction, like that seen in Figure 1 (see Goyal et al. [1991] for a discussion of such friction models). While some materials do have friction of this type, such anisotropic friction models are more generally useful as a means to approximately model compliance effects and wheel tread effects. For instance, if the wheel shown above was as thin as a saw blade, then we would expect $\mu_{R}$ (the friction coefficient along the "rim" direction) to be less than $\mu_{S}$ (the friction coefficient along the "side" direction), and in doing so we would be modeling the nonideal point contact in terms of a variable coefficient of friction. However, the treads on a tank ensure that $\mu_{R}$ is greater than $\mu_{S}$. Note that the minimum of the dissipation function will only be non-unique when the ellipse reduces to a circle (i.e. $\mu_{S}=\mu_{R}$ ). Moreover, the same indeterminacy shows up in the analogous Lagrangian analysis.

\subsection{XY stability and $\theta$ instability}

In Murphey and Burdick [2001] we showed that when on takes the contact mechanics and discretness of the actuatory array into account, the orientation $\theta$ of the moving object is unstable at the equilibrium configuration when one uses the programmable vector field approach. First we note that the above system is a differential inclusion of the type found in Filippov [1988]. I.e. due to the switching of the control vector fields in Eq. (4), the governing equations take the form $\dot{x} \in F(t, x)$. For our analysis we will require the following theorem from Filippov [1988]. We remind the reader that the upper and lower derivatives for a function $V(t, x) \in C^{1}$ are defined by:

$$
\dot{V}^{*}=\sup _{y \in F(t, x)}\left(V_{t}+\nabla V y\right) \quad \dot{V}_{*}=\inf _{y \in F(t, x)}\left(V_{t}+\nabla V y\right)
$$

Theorem 3 below from Filippov [1988] is the generalization of time varying Lyapunov theory to differential inclusions.

Theorem 3 Let, in a closed domain $D\left(t_{0} \leq t<\infty,|x| \leq\right.$ $\left.\epsilon_{0}\right)$, the differential inclusion $\dot{x} \in F(t, x)$ satisfy the basic conditions of existence and $0 \in F(t, 0)$; in this domain, let there exist functions $V(t, x) \in C^{1}, V_{0}(x) \in C$ for which

$V(t, 0)=0, \quad V(t, x) \geq V_{0}(x)>0 \quad\left(0<|x|<\epsilon_{0}\right)$ Then:

1) If $\dot{V}^{*} \leq 0$ in $D$, the solution $x(t)=0$ of the inclusion $\dot{x} \in F(t, \bar{x})$ is stable.

2) If, moreover, there exist functions $V_{1}(x) \in C, W(x) \in$ $C\left(|x| \leq \epsilon_{0}\right)$ and

$$
\begin{aligned}
0<V_{0}(x) \leq V(t, x) & \leq V_{1}(x), \\
\left(0<|x|<\epsilon_{0}\right), & \dot{V}^{*} \leq-W(x)<0,
\end{aligned}
$$

then the solution $x(t)=0$ is asymptotically stable.
In our generalization we assume that all the actuators are a finite distance apart and make point contact with the object being manipulated. The following theorem indicates that the induced instability of the programmable vector field approach can arise in more general circumstances. The proof of the theorem relies essentially upon book-keeping the conditions found in Theorem 3, and can be found in Murphey and Burdick [2001].

Theorem 4 Given an elliptic vector velocity field $\Psi(x, y)$ : $\mathbb{R}^{2} \rightarrow \mathbb{R}^{2}$, and a discrete planar array geometry in some neighborhood of the origin, the solution to the kinematics given by the PDM is stable in $(x, y)$ and unstable in $\theta$. Moreover, if $\mu_{S}<\mu_{R}$, then such a system is stabilizable through a discontinuous feedback law.

Now the $\mu_{S}<\mu_{R}$ assumption only has an obvious physical interpretation for wheel-like contacts, and we do not yet have a formulation for generic contacts. The case $\mu_{R}<\mu_{S}$ may be stabilizable, but not in as straight forward a fashion.

\section{The Main Result}

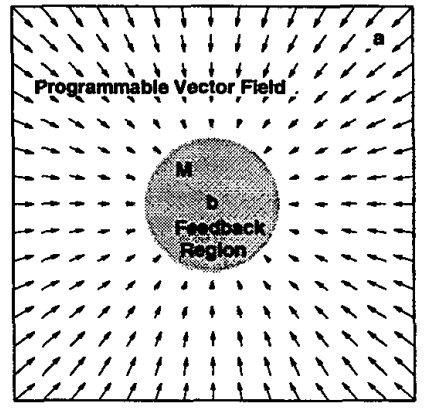

Figure 2: A LASALLE Invariance Theorem

Here we "blend" the philosophies of Sudsang and Kavraki [2001], Böhringer et al. [2000] and Murphey and Burdick [2001] using a variation of the classical LaSalle Invariance Principle (see LaSalle [1968]). I.e., we use the programmable vector field approach to govern the gross motions of the object far away from the equilibrium point, and our local stablizing feedback law Murphey and Burdick [2001] in the vicinity of the equilibrium configuration. The intuition behind this result, and its application to the problem at hand, is that if we can move a package from one point $a$ in the plane to another point $b$ (an equilibrium point), and if we have feedback in a neighborhood of point $b$, we can allow the package to spin freely along its path to $b$, and wait to concern ourselves with the package's orientation after it has come sufficiently close to $b$. Consider Fig. 2. If $a$ is in the upper right hand corner, then it is clear that even with switching between contact states, a package starting at $a$ will eventually arrive in the feedback region $\mathcal{M}$ in the middle of Fig. 2. In fact, Theorem 4 implies just that, since translation motions (but not rotational motions) are stable under the programmable vector field model, even when discrete contacts are taken into account. From a practical point 
of view this means that as long as we have no performance goals for the orientation $\theta$ outside of $\mathcal{M}$, we do not need feedback outside of $\mathcal{M}$. The goal of this section is to formally prove these intuitive notions. However, because of the multi-model aspect of our governing equitions, we must extend Lasalle's theorem.

We should note that the basic difference between the classical version of the LaSalle theorem and ours is that we must consider systems goverened by differential inclusions. In such systems, the idea of a "flow" does not necessarily include uniqueness. That is, rather than having a result good for the flow $\phi(t)$, we must have it for any flow $\phi(t)$ satisfying $\dot{\phi} \in F(t, x)$. Indeed, this is the underlying theme to much of the study of stability of differential inclusions.

Theorem 5 Let $\mathcal{M}$ be the "feedback region," a compact simply connected subset of $\mathbb{R}^{2}$. Let $V(x)$ be a Lyapunov function on $\mathcal{M}$. Let $\phi\left(t, x_{0}\right)$ denote a flow that satisfies $\dot{x} \epsilon$ $F(x, t)$, starting from $x_{0}$. Let $\mathcal{M}$ be a positively invariant compact set under all flows $\phi(t, p)$ satisfying the differential inclusion $\dot{x} \in F(x, t)\left(\mathcal{M}\right.$ is positively invariant if $\dot{V}^{*}(x) \leq$ 0 for all $x \in \mathcal{M}$, where $\dot{V}^{*}$ is defined in (5)). Now let

$$
\begin{aligned}
& E=\{x \in \mathcal{M} \mid 0 \in \dot{V}(x)\} \\
& N=\left\{\bigcup \phi\left(t, x_{0}\right) \mid x_{0} \in E \text { and } \phi(t) \in E \forall t>0\right\}
\end{aligned}
$$

(I.e. $E$ is the set on which the Lyapunov function is zero, and $M$ is the union of all trajectories that start in $E$ and remain in $E$ for all $t>0$ ) Then, for all $x \in \mathcal{M}, \phi(t, x) \rightarrow N$ as $t \rightarrow \infty$.

Proof: This proof is roughly patterned on the proof of LaSalle's Invariance Theorem found in Wiggins [1990]. First we recall that the $\omega$-limit point of a differential inclusion (or differential equation) and a point $p \in \mathbb{R}^{n}$ is defined as a point $q \in \mathbb{R}^{n}$ where for all solutions $\phi_{t}(p)$ to the differential inclusion $\dot{x} \in F(x, t) \exists t_{1}, \ldots, t_{i}$ with $i \uparrow \infty$ such that $\phi\left(t_{i}\right) \rightarrow q$ as $i \uparrow \infty$. The $\omega$-limit set is the collection of such points, and is denoted $\omega(p)$.

Now we need to show that $V=0$ on $\omega(p)(\forall p \in \mathcal{M})$. Assume $q$ is an $\omega$-limit point of the differential inclusion, then set $V(q)=k$. Now we will show that $V$ is constant on $\omega(p)$. First we will need the following fact about $\omega$-limit sets.

Lemma $1 \omega(p)$ is invariant under the flow of $F$.

Proof: Let $q \in \omega(p)$ and $q_{s}=\phi_{s}(q)$. We first must consider if the map $\phi_{s}(\cdot)$ exists for all $s$ ? First, note that since $\mathcal{M}$ is compact, we have existence of $\phi_{s}(\cdot)$ for $s \in(0, \infty)$. (This is a natural extension of the classical result for ODE's - see [Filippov, 1988, pages 77-86]) Now we show that it is true for $s \in(-\infty, 0)$. Using the fact that the limit of any uniformly convergent sequence of solutions to a differential inclusion is also a solution (see Lemma 1 in [Filippov, 1988, page 76]), we can choose a sequence $\left\{t_{i}\right\}$ with $t_{i} \rightarrow \infty$ as $i \uparrow \infty$ such that $\phi_{t_{i}}(p) \rightarrow q$ as $i \uparrow \infty$. (this is by definition of $\omega(p))$ Then using the fact that $\phi_{s}\left(\phi_{t_{i}}\right)=\phi_{s+t_{i}}($ a.e.) as one takes the limit $i \uparrow \infty$, we get that $\phi_{s}(q)$ exists for $s \in(-\infty, 0)$.
Now that we have the existence of the map $\phi_{s}(\cdot)$ for all $s$, we can choose a sequence $t_{1}, \ldots, t_{i} i \uparrow \infty$ such that $\phi_{t_{i}} \rightarrow q$ as $t \rightarrow \infty$. Then the map $\phi_{t_{i}+s}(p)=\phi_{s}\left(\phi_{t_{i}}(p)\right)$ converges to $q_{s}$ as $i \uparrow \infty$. This implies that $q_{s} \in \omega(p)$ and $\omega(p)$ is therefore invariant.

Now this implies that $k=\inf \left\{V\left(\phi_{t}(x)\right) \mid t \geq 0\right\}$ because $\dot{V} \leq 0$ everywhere in $\mathcal{M} . k=\inf \left\{V\left(\phi_{t}(x)\right) \mid t \geq 0\right\}$ implies $V\left(\phi_{t}(x)\right)=k$, so $\dot{V}=0$ on $\omega(p)$. This in turn implies that $\omega(p) \subset E$. Again, because of the above fact that $\omega(p)$ is invariant, $\omega(p) \subset N$. This in turn implies that $\phi_{t}(x) \rightarrow N$ as $t \rightarrow \infty$, the desired result. $\square$

Now to apply this to the case of distributed manipulation, we must only show that a distributed manipulator will satisfy the requirements and assumptions of Theorem 5. This will lead to the following Corollary of Theorem 5. Assume the distributed system can be represented by an array of actuators $a_{i j}$ with the coordinate location of $\left(x_{i}, y_{j}\right)$, and assume that the PDM model solution depends only on the center of mass. (Equivalently, that the coefficient of friction is uniform) For us, $\mathcal{M}$ will be the feedback region of the distributed manipulator, that is, the area in which we have some sort of state feedback available to us.

Corollary 6 Given a discrete planar array geometry, an elliptic vector velocity field $\Psi(x, y): \mathbb{R}^{2} \rightarrow \mathbb{R}^{2}$ outside of $\mathcal{M}=B_{\epsilon} \times S_{1}$ for some $\epsilon>0$, and a locally stabilizing feedback law (such as the one in Theorem 4) the solution to the kinematics given by the PDM is globally stable.

Proof: We assume that the desired equilibrium point is always in $\mathcal{M}$. This implies that since $\mathcal{M} \subset S E(2)$, then $\mathcal{M}=B_{\epsilon} \times S_{1}$ where $B_{\epsilon}$ is the $\epsilon$-ball in $\mathbb{R}^{2}$, and $\mathcal{M}$ is therefore compact. Therefore the first part of Theorem 5 is supplied. $\mathcal{M}$ is positively invariant by Theorem 4 using an elliptic vector field. Moreover, for a choice of $V=\|q\|^{2}$, $E$ consists solely of the origin. This implies that the origin is stable. In fact, asymptotically stable, because $\mathcal{M}$ is reached in finite time, and the once inside $\mathcal{M}$ the origin is asymptotically stable by Theorem 4 .

\section{Simulations}

To illustrate these concepts, in this section we provide the results of simulations that model a distributed manipulation system with actuatuators located at $(i, j)$ in the plane, with feedback only within a region $\mathcal{M}=B_{\mathrm{r}=4}$, that is only within a region of radius 4 (in the length units used by the simulation). We used Mathematica to do these simulations, using its NDSolve integrator, modified to allow for differential inclusions. Some modification is necessary in order to avoid the numerical difficulties of approaching a switching boundary (in the case of these simulations $y=x, y=-x, x=0, y=0$ ). However, this is only a concern for switching boundaries which are stable or attracting, because if the trajectory intersects the boundary transversely standard numerical schemes still work. For these simulations, we allowed the switching boundaries which were stable to have the averaged, projected dynamics. This, like 
the method of introducing hysteresis to simulate a discrete system as a hybrid automaton, produces numerically stable simulations. We should point out, however, that the choice of the averaged solution is only one possible choice satisfying the differential inclusion. That is, if we have a boundary $N$ and kinematics $g_{\sigma_{1}}$ on one side and $g_{\sigma_{2}}$ on the other, the choice of $\dot{q}=\frac{g_{\sigma_{1}}+g_{\sigma_{2}}}{2}$ is just one choice satisfying $\dot{q} \in F=\operatorname{co}\left\{g_{\sigma_{1}}, g_{\sigma_{2}}\right\}$. The main difficulty is that solutions of differential inclusions are necessarily nonunique, therefore implying that any simulation represents only one solution $\phi$ to the differential inclusion $\dot{\phi} \in F$. Fig. 3 shows a box of unit mass being moved from $\left\{3,10, \frac{\pi}{4}\right\}$ to the origin in $\mathbb{R}^{2}$ over wheels with constant friction coefficient $\mu$ and then stabilized to $\theta=0$, where $\theta$ is measured between the box's long axis and the $x$ axis of $\mathbb{R}^{2}$. The actuators are assumed to be at $( \pm 2 i, \pm 2 j)$, with point contact between the wheel and the box. The wheels are unit radius. While outside $\mathcal{M}$ the programmable vector field used was simply $\{\dot{x}, \dot{y}\}=\{-x,-y\}$. Inside $\mathcal{M}$ we use the feedback law derived from Theorem 4 to stabilize the box to the origin. Controls that succeed in stabilizing everything in the $\mathcal{M}$ are $u_{i}=|k \theta|+k|(x, y)|$ and $u_{j}=-k \theta$, where $u_{i}$ is the control associated with the first two constraints determining the kinematics as predicted by the PDM, and $u_{j}$ is the control associated with the third constraint determining the kinematics as predicted by the PDM. Snapshots of the box were taken for every time $t=1, \ldots 12$. The lines on the simulation represent the different nodes as the package crosses them (one should think of these nodes as covering the plane). The box in the simulation represents a portion of the box being transported. This was done so that the simulation could be visualized more easily. The four nodes inside the circle represent the four node system studied in Murphey and Burdick [2001].

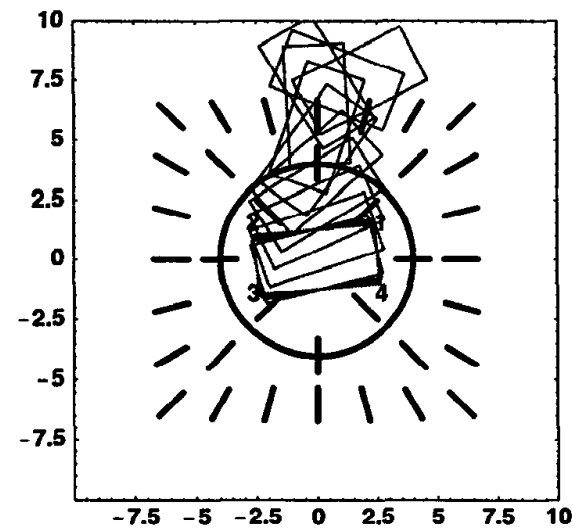

Figure 3: A Box Being Transported to $\{x, y, \theta\}=\{0,0,0\}$ from $\left\{3,10, \frac{\pi}{4}\right\}$

\section{Conclusions}

Here we found that the locally stabilizing aspect of the controller obtained in Murphey and Burdick [2001] can be successfully used in conjunction with the global method of programmable vector fields to provide global stability without requiring global feedback. This has consequences not only for simplicity of design, but also simplicity of implementation. Despite the need for local feedback, there is no reason to have to incorporate the costly addition of feedback throughout the path followed by the object. Our current work includes building an experimental setup using wheeled actuators and visual feedback to validate the theory provided both in this and other work.

Acknowledgements: This work was partially supported by a grant from the National Science Foundation (grant NSF9402726) through its Engineering Research Center (ERC) program.

\section{References}

J.C. Alexander and J.H. Maddocks. On the kinematics of wheeled vehicles. The International Journal of Robotics Research, 8(5): 15-27, October 1989.

K.F. Böhringer, B. Donald, and N. MacDonald. Upper and lower bounds for programmable vector fields with applications to mems and vibratory plate parts feeders. In Algorithms for Robotic Motion and Manipulation, pages 255-276. A.K. Peters, Ltd., 1997.

K.F. Böhringer, B.R. Donald, L.E. Kavraki, and F. Lamiraux. A distributed, universal device for planar parts feeding: unique part orientation in programmable force fields. In Distributed Manipulation, pages 1-28. Kluwer Academic Pub., 2000.

M.A. Erdmann and M.T. Mason. An exploration of sensorless manipulation. IEEE Journal of Robotics and Automation, 4(4), 1988.

A.F. Filippov. Differential Equations with Discontinuous Right Hand Sides. Kluwer Academic Publishers, 1988.

K.Y. Goldberg. Orienting polygonal parts without sensing. Algorithmica, 143(2/3/4):201-225, 1993.

S. Goyal, A. Ruina, and J. Papadopoulos. Planar sliding with dry friction. WEAR, 143:307-352, 1991.

J.P. LaSalle. Stability theory for ordinary differential equations. $J$. Diff. Eq., 4:57-65, 1968.

T. D. Murphey and J. W. Burdick. Issues in controllability and motion planning for overconstrained wheeled vehicles. In Proc Int Conf Math Theory Networks Systems (MTNS), Perpignan, France, 2000.

T. D. Murphey and J. W. Burdick. On the stability and design of distributed systems. In Proc. IEEE Int. Conf. on Robotics and Automation, Seoul, Korea, 2001.

A. Sudsang and L. Kavraki. A geometric approach to designing a programmable force field with a unique stable equilibrium for parts in the plane. In Proc. IEEE Int. Conf. Robotics and Automation, Seoul, Korea, 2001.

Wiggins. Introduction to Applied Nonlinear Dynamical Systems and Chaos. Springer Verlag, 1990. 